\title{
Analysis of the integration of the three-way catalyst thermal management in the on-line supervisory control strategy of a gasoline full- hybrid vehicle
}

\author{
Marco Benegiamo ${ }^{1}$, Paolo Carlucci ${ }^{1}$, Vincenzo Mulone ${ }^{2}$, and Andrea Valletta $^{1, *}$ \\ ${ }^{1}$ Università del Salento, Piazza Tancredi, 7, 73100 Lecce, Italy \\ ${ }^{2}$ Università di Roma Tor Vergata, via del Politecnico 1, 00133 Rome, Italy
}

\begin{abstract}
Full hybrid electric vehicles have proven to be a midterm viable solution to fulfil stricter regulations, such as those regarding carbon dioxide abatement. Although fuel economy directly benefits from hybridization, the use of the electric machine for propulsion may hinder an appropriate warming of the aftertreatment system, whose temperature is directly related to the emissions conversion efficiency. The present work evaluates the efficacy of a supervisory energy management strategy based on Equivalent Minimization Consumption Strategy (ECMS) which incorporates a temperature-based control for the thermal management of the Three-Way Catalyst (TWC). The impact of using only the midspan temperature of TWC is compared against the case where temperature at three different sampling points along the TWC length are used. Moreover, a penalty term based on TWC temperature has been introduced in the cost functional of the ECMS to allow the control of the TWC temperature operating window. In fact, beyond a certain threshold, the increase of the engine load, requested to speed up TWC warming, does not translate into a better catalyst efficiency, because the TWC gets close to its highest conversion rate. A gasoline P2 parallel full hybrid powertrain has been considered as test case. Results show that the effects of the different calibrations strategies are negligible on the TWC thermal management, as they do not provide any improvements in the fuel economy nor in the emissions abatement of the hybrid powertrain. This effect can be explained by the fact that the charge sustaining condition has a greater weight on the energy management strategy than the effects deriving from the addition of the soft constraints to control the TWC thermal management. These results hence encourage the use of simple setups to deal with the control of the TWC in supervisory control strategies for full hybrid electric vehicles.
\end{abstract}

\footnotetext{
* Corresponding author: andrea.valletta@unisalento.it
} 


\section{Introduction}

The share of hybrid and electric vehicles in the worldwide market has increased in recent years to meet the stringent regulations concerning vehicle pollution [1], also thanks to the decrease of the their total cost of ownership [2]. Hybrid technologies for vehicle propulsion are a bridging solution towards fully electrified zero emission vehicles which will gradually play an important role in future mobility as issues concerning electric energy storage technologies and distribution are solved such as the charging speed regarding the usage of batteries or the extraction of raw materials when considering their manufacturing [3].

Nowadays the most common hybrid powertrains are powered by gasoline engines and this can be mainly explained by the fact that they are subject to lower exhaust emission content with respect to compression-ignition diesel engines, hence requiring less complex aftertreatment systems and so they are able to provide overall contained costs when hybridization of the powertrain is performed.

Hybrid electric vehicles are characterized by a degree of electrification ranging from micro-hybrid vehicles to full-hybrid ones [4]. There exist also different powertrain architectures, such as the series, the parallel and the series-parallel (also called power-split) that allow to change the number of degrees of freedom to operate the vehicle by using the internal combustion engine (ICE) and the electric machine/s (EM) at the same time [5]. An additional classification is based on the possibility to externally recharge the battery (i.e. home socket): in this case, a hybrid vehicle is referred to as plug-in hybrid (PHEV). PHEV powertrains have a greater capability to operate using the electrical energy, even though they require a higher level of electrification with respect to full-hybrid vehicles (FHEV). In terms of control, hybrid vehicles require a supervisory energy management control which is a higher-level control with respect to single components controls (i.e. engine control unit (ECU) and battery management system (BMS)). Supervisory energy management control relies on sophisticated algorithms which manage the power split between the internal combustion engine (ICE) and the electric machine/s (EM) and at the same time guarantee the best energy use to ensure the minimum fuel consumption.

FHEV and PHEV powertrains are characterized by different strategies: the former use the so-called charge-sustaining strategies as the battery energy must be preserved onboard, while the latter use a combination of charge-depleting and charge-sustaining strategy. In the past two decades, scientific literature was enriched by studies concerning different approaches to the control of the hybrid powertrains. Supervisory energy management strategies can be mainly classified in rule-based strategies and optimal control strategies. The advantage of optimal control strategies is the possibility to determine the power split between ICE and EM by solving an optimization problem where the cost function can be suitably modeled without embarking onto difficult control design as in the former case. Besides Dynamic Programming (DP), which returns the global optimal solution if the future state of the powertrain is known, Equivalent Consumption Minimization Strategy (ECMS) has proven to be a convenient solution when computational cost and real-time applicability are considered. Moreover, ECMS can be adapted in order to adjust the power-split between the ICE and EM according to feedback signals such as the battery state of charge (SoC) or the driving cycle related data, leading to results similar to the ones that can be obtained by more sophisticated model predictive control [6].

A charge-depleting strategy may require frequent start-stops of the engine so that, especially concerning the aftertreatment system efficiency, this could be detrimental when the temperature of the converter is below the light-off temperature. A classification of strategies that consider the emissions into the optimization problem is reported in [7]: generally pollutants mass flow rates are incorporated into the cost function of the optimization problem of the supervisory energy management control strategy. Moreover a 
recent example of this implementation for a hybrid parallel powertrain can also be found in the work of Guille des Buttes et al. [8]. In a previous work [9], the Authors demonstrated how the integration of the three-way-catalyst (TWC) thermal management into the supervisory energy management control strategy, based on ECMS algorithm, is beneficial in terms of TWC operation by reducing the time to reach the light-off temperature. However, at an early stage of development, the midspan temperature of the TWC converter was used as the additional state variable to the control strategy algorithm to guarantee a suitable TWC warming. This choice was motivated to make a fair comparison with other studies found in literature as well as to not overcomplicate the control strategy.

In the present work, a thorough analysis is made regarding the effects of different calibrations of the ECMS supervisory energy management control strategy which incorporates the TWC thermal management. More specifically, two aspects are covered: the first is a sensitivity study to investigate how some parameters dealing with TWC thermal management affect the output of the strategy in terms of fuel consumption and emission reduction; the second leverages the use of 1D TWC model to understand whether additional temperature sampling points along the TWC axis, beside middle section, can significantly improve the TWC thermal management. The analysis is carried through numerical simulation of the P2 parallel hybrid electric vehicle over the World-wide harmonized Light-duty Test Cycle (WLTC).

\section{Materials and methods}

\subsection{Numerical Simulation Setup}

The present study was performed by means of numerical simulations. A conventional gasoline passenger vehicle, equipped with a TWC, as well as the $48 \mathrm{~V}$ battery package and the electric machine were tested at "Centro Studi Componenti per Veicoli S.p.A" Bosch $\mathrm{GmbH}$ facility. For this reason, all the characteristic data used to build the numerical model is hidden for confidentiality. Nevertheless, this does not compromise the outcome of the presented work which has the goal to show the influence of different calibrations of the ECMS control strategy oriented to TWC thermal management on the vehicle performance.

The numerical model of the supervisory energy management control strategy was developed in MATLAB-Simulink, while the powertrain model was built in GT-Suite. The control code was then paired to GT-Suite as represented in Fig. 1: all the control signals which result from the on-line optimization process carried out by the ECMS-based algorithm are sent to GT-Suite powertrain model, which sends back to the control strategy the powertrain components state signals, such as the battery SoC, the ICE actual output torque and the TWC temperature at the used sampling points.

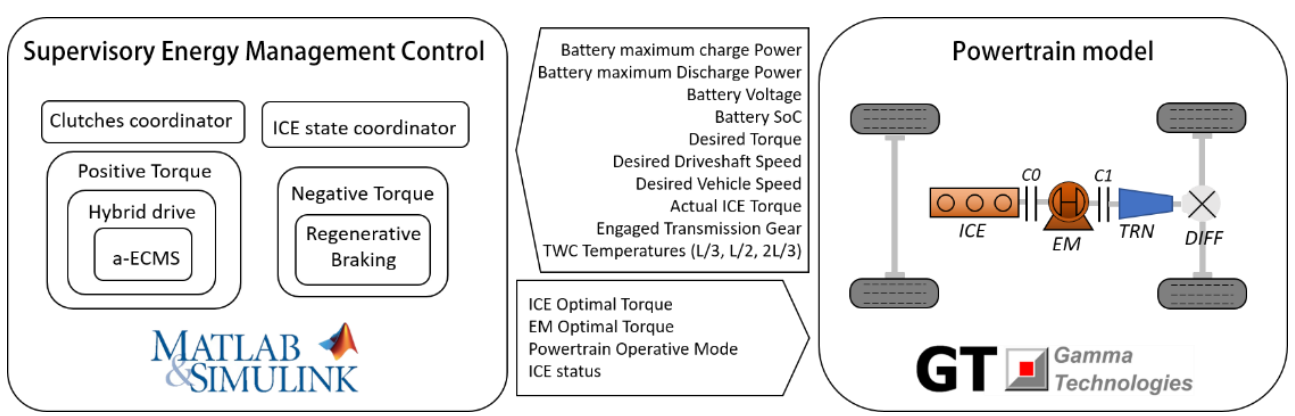

Fig. 1. Schematic representation of the P2 HEV model: supervisory energy management strategy is built in MATLAB-Simulink; the powertrain model is built in GT-Suite 
The hybrid vehicle performance is tested over the WLTC driving cycle, which is made of four distinctive driving phases, as represented in Fig. 2: urban (low), suburban (medium), rural (high) and highway (extra high). The modelling of the powertrain components is explained in the previous work of the Authors [9]. In particular, the ICE and EM were modelled with a map-based approach derived through calibration process at the test-bench.

The 48V battery is modelled with a simple internal-resistance circuit which was calibrated with data recorded during experimental tests, such as: the relationship between open-circuit voltage, state of charge and battery mean temperature; the battery current limitations that are implemented in the model with an empirical relation, which depends on the electrical energy throughput. The thermal behavior of the TWC model was characterized with data collected during vehicle dynamic tests, while the chemical kinetics was modelled according to the work of Ramanathan et al. [10]

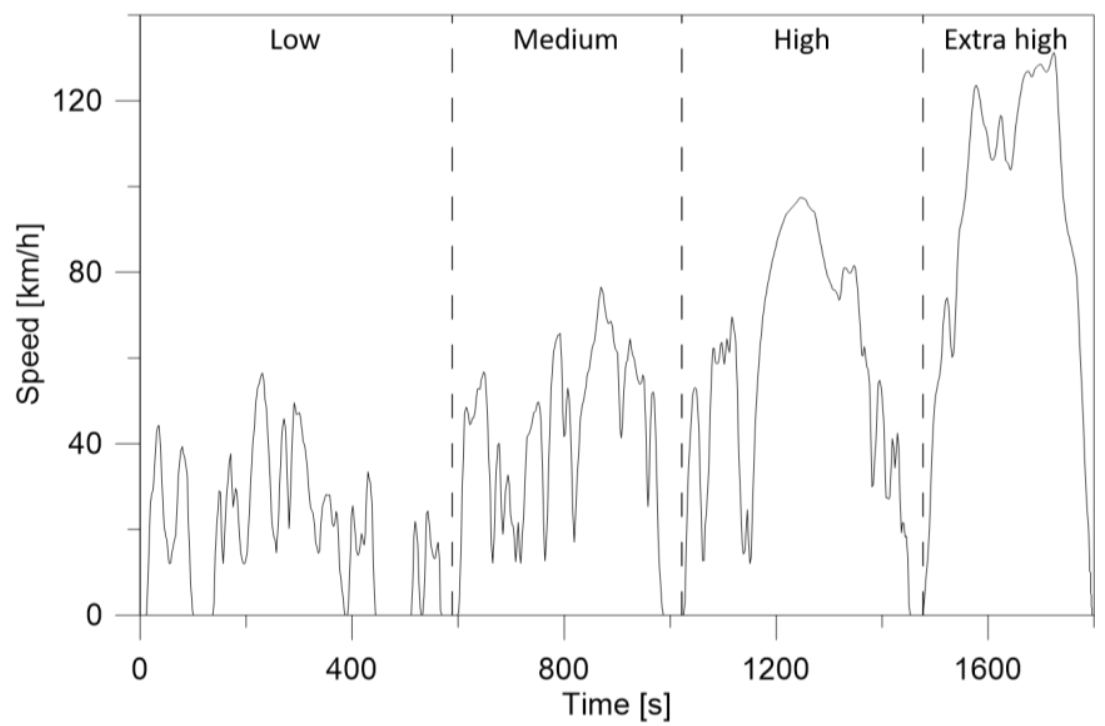

Fig. 2. World-wide harmonized Light-duty Test Cycle (WLTC)

\subsection{Adaptive Equivalent Consumption Minimization Strategy with TWC Thermal Management}

The Equivalent Consumption Minimization Strategy was developed over two decades ago by Paganelli et al. [11] intuitively and later analytically demonstrated with Pontryagin Minimum Principle. On the contrary of global optimization algorithms such as DP [12], ECMS solves an instantaneous optimization problem without any a-priori knowledge of the future state of the system and for this reason can only provide a sub-optimal solution for the entire time horizon of the problem. For the control of a FHEV powertrain, an instantaneous optimization problem is solved to determine the best control of the EM and ICE of the hybrid vehicle so to obtain a minimum fuel consumption while satisfying the charge-sustaining condition. The battery is a buffer of energy suitably stored and conveniently used to propel the vehicle and to allow the engine to operate more efficiently, this resulting in fuel economy improvement.

This concept is put into practice by minimizing at each time instant a cost function which is written in terms of an equivalent fuel consumption (equation 1):

$$
\dot{m}(u(t), t)_{f u e l, e q v}=\dot{m}(u(t), t)_{f u e l, I C E}+s(t) * \dot{m}(u(t), t)_{f u e l, R E E S}
$$


where $u(t)$ is the control variable (for example it can be the ICE torque output), $\dot{m}_{f u e l, I C E}$ is the fuel spent by the ICE to propel the vehicle, the second term on the right-hand side of the equation, $\dot{m}_{f u e l, R E E S}$, is the fuel spent by the ICE to recharge the battery and possibly keep its energy content constant and it depends on the current use of the electrical energy of the battery by the electric machine. The equivalence is enabled by the so-called equivalence factor $s(t)$, which is a key parameter in the calibration of the control strategy. For convenience of implementation into the supervisory control, equation 1 can be expressed in terms of powers, by multiplying both sides of the equation by the fuel low heating value $Q_{L H V}\left[\frac{J}{k g K}\right]:$

$$
P_{\text {fuel, }, \text { qv }}=P_{\text {fuel,ICE }}+s(t) * P_{\text {fuel, REES }}
$$

The previous cost function used by ECMS control strategy can be derived analytically starting from the cost function of the global optimization problem (equation 3):

$$
J(u(t))=\int_{0}^{t_{f}}\left[Q_{L H V} \dot{m}_{f u e l}(u(t), t)+\alpha\left(\frac{\operatorname{SoC}_{r e f}-\operatorname{SoC}(t)}{\Delta S o C_{\text {norm }}}\right)^{2 a}\right] d t
$$

where a penalty is added to constrain the SoC deviation around a reference SoC value, $S o C_{r e f}$, within an admissible range $\Delta$ SoCnorm $(0.4-0.8) . \alpha$ is a weight factor and a the order of the penalty. The instantaneous optimization problem is derived by extracting the Hamiltonian function from equation 3:

$$
H(\operatorname{SoC}, u(t), t)=Q_{L H V} \dot{m}_{f}(u(t), t)+\alpha\left(\frac{\operatorname{SoC}_{r e f}-\operatorname{SoC}(t)}{\Delta \operatorname{SoC} C_{\text {norm }}}\right)^{2 a}+\lambda(t) \operatorname{So} C
$$

where the costate $\lambda(t)=-s(\operatorname{SoC}(t), t) U_{O C}(\operatorname{SoC}(t)) E_{B a t t}$. This brings to an equivalent expression for equation 2. If the optimal cost-to-go function $J^{*}(\operatorname{SoC}, t)$, associated to $J$, were known, the optimal costate could be calculated according to Hamilton-Jacobi-Bellman theory:

$$
\lambda^{*}(S o C, t)=\frac{\partial J^{*}(S o C, t)}{\partial S o C}
$$

Equation 5 can however be only calculated through an estimation of $J^{*}$, which is not known a-priori. According to the work of Ambhul [13] the equivalence factor can then be written as in equation 6 , which gives the instantaneous cost function to be solved for a fuel-economy oriented control strategy:

$$
s(t)=s_{0}+\int_{0}^{t} \frac{S o C_{r e f}-\operatorname{SoC}(\tau)}{T_{i}} d \tau+\tilde{\alpha} \frac{\left(\operatorname{SoC} C_{r e f}-\operatorname{SoC}(t)\right)^{2 a-1}}{U_{O C}(\operatorname{SoC}) E_{\text {batt }}}
$$

where $s_{0}$ is a constant parameter, $T_{i}$ is the time of the integral term, $\tilde{\alpha}$ derives from the linear approximation of the SoC trajectory over a given time, $U_{O C}$ is the battery open-circuit voltage and $E_{\text {batt }}$ the battery nominal capacity. A strategy in this sense would try to minimize the fuel consumption by preferring the use of the electric machine whenever the use of the ICE is less efficient, for example during low vehicle speed operation. Moreover, the supervisory control strategy could provoke too frequent ICE shutoffs with a possible decay in aftertreatment performance, especially if the light-off temperature has not been yet reached. For this reason, the cost function used in the optimization problem could be extended by integrating the thermal management of TWC. The TWC state variable adopted in this work is the catalyst temperature $\vartheta_{T W C, i}$ measured at a point "i" on the axis of symmetry (assuming a cylindrical shape for the TWC). Moreover, the formulation can be generalized by including three sampling points for the temperature, at $\frac{1}{3}, \frac{1}{2}$ and at $\frac{2}{3}$ of the length $L$ and a weighted sum 
( $w_{i}$ is the generic weight factor) is used to take them into account so to use only one additional costate variable $\rho$, apart from $s$.

$$
\begin{aligned}
H(u, t) & =P_{\text {fuel }}(u, t)+s P_{\text {batt }}(u, t)+\alpha\left(\frac{\operatorname{SoC} C_{\text {ref }}-\operatorname{SoC}(t)}{\Delta \operatorname{So} C_{\text {norm }}}\right)^{2 a} \\
+k & \left(\sum_{i} w_{i} \vartheta_{T W C, i}\right) \sum_{i} w_{i} \vartheta_{T W C, i}+\rho \sum_{i} w_{i} \dot{\vartheta}_{T W C, i}
\end{aligned}
$$

The sum is computed for the different temperature sampling points used: in this work three sampling points along the TWC axis are used $\frac{L}{3}, \frac{L}{2}, \frac{2 L}{3}$. Similarly to the fuel economy oriented formulation, the fourth term in equation 7 is a penalty term that is added to penalize all candidate solutions that do not use the ICE when the TWC temperature is below the lightoff temperature $T_{T W C}^{L O}$ and that use the ICE when the TWC temperature is above the admissible maximum limit $T_{T W C}^{u p}$. The penalty factor $k$ is calculated according to Fig. 3, based on the weighted sum of the temperatures at the chosen sampling points along the TWC axis. The value of the penalty beyond $T_{T W C}^{u p}$ can be suitably varied with a gradient value $g$.

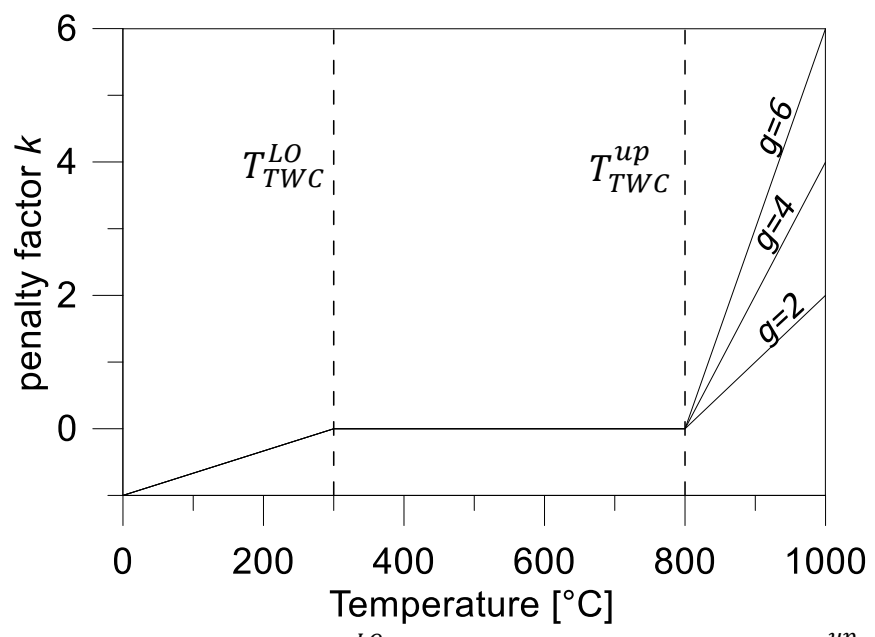

Fig. 3. TWC temperature penalty factor k: $T_{T W C}^{L O}$ is the light-off temperature and $T_{T W C}^{u p}$ the temperature upper limit, $\mathrm{g}$ is the gradient value for the penalty beyond $T_{T W C}^{u p}$

In the first analysis presented in this work, the value of $T_{T W C}^{u p}$ is set to three different values $\left(400,600,800{ }^{\circ} \mathrm{C}\right)$. The objective is to benefit from the good TWC conversion efficiency already at a temperature of $500{ }^{\circ} \mathrm{C}$ and hence to penalize a further usage of the ICE by activating the penalty term $k$, which can be also calibrated with different gradient values. Moreover, although new TWC technologies enable high operating temperatures in the range of $1000^{\circ} \mathrm{C}$ while ensuring good conversion efficiencies, the catalyst aging worsens at high temperatures and already in the range of $900{ }^{\circ} \mathrm{C}$ alumina sintering may occur [14], so that it would be ideal to control the maximum temperature reached by the TWC.

\section{Results and discussion}

This section is divided into two parts where each one independently analyzes a calibration aspect of the proposed strategy in order to understand its impact on the TWC thermal management and on the overall energy management strategy: the first one is to investigate the effect of different tunings of the TWC temperature penalty term, explained in equation 7; 
the second one focuses on understanding the impact of the addition of temperature sampling points along the TWC axis to the cost function formula, apart from the one used at the midspan. It is important to note that the control strategy was calibrated every time so to have a maximum deviation of the final battery SoC value from the target value $S o C_{r e f}$ equal to $\pm 1 \%$ : this enables a fair comparison between different calibration setups in terms of fuel consumption and emissions, since the net battery energy balance is almost null.

\subsection{Calibration of the Penalty Term}

Two quantities define the penalty of the TWC desired temperature operational window, illustrated in Figure 3: the upper boundary of the zero-plateau $T_{T W C}^{u p}$ and the slope of the segment beyond it. The sensitivity analysis is carried out for 3 values of $T_{T W C}^{u p}=$ $\{400,600,800\}^{\circ} \mathrm{C}$ and the gradient value of the penalty factor $k, g=\{2,4,6\}$.

For a comprehensive overview of the simulated nine cases, the results are compared by looking at the most representative data collected in Table 1: the TWC maximum temperature $T_{T W C}^{\max }$ evaluated for the low and medium parts of the WLTC, the time needed to reach the light-off temperature $T_{T W C}^{L O}$ as well as the fuel consumption and the TWC conversion efficiencies over the driving cycle, which are expressed in percentage deviation with respect to the baseline case $T_{T W C}^{u p}=800^{\circ} \mathrm{C}$ and gradient value equal to 2 .

Table 1. Characteristic results of the TWC performance for different tunings of the temperature penalty term. Fuel consumption and conversion efficiencies are referred to the case highlighted in bold

\begin{tabular}{c|c|ccc|ccc|ccc}
\multicolumn{1}{l|}{$T_{T W C}^{u p}$} & {$\left[{ }^{\circ} \mathrm{C}\right]$} & \multicolumn{3}{|c|}{400} & \multicolumn{3}{|c}{600} & \multicolumn{3}{|c}{800} \\
\hline Gradient value & {$[-]$} & 2 & 4 & 6 & 2 & 4 & 6 & 2 & 4 & 6 \\
\hline $\begin{array}{c}\left.T_{T W C}^{\text {max }}\right)_{W L T C-l o w} \\
\left(T_{T W C}^{\text {max }}\right)_{W L T C-\text { medium }}\end{array}$ & {$\left[{ }^{\circ} \mathrm{C}\right]$} & 720 & 725 & 726 & 724 & 727 & 729 & 730 & 727 & 730 \\
Time to $T_{T W C}^{L O}$ & 761 & 730 & 746 & 749 & 771 & 755 & 732 & 744 & 738 \\
$\Delta F C$ & {$[\mathrm{~s}]$} & 31 & 31 & 31 & 31 & 31 & 31 & 31 & 31 & 31 \\
$\Delta \eta_{N O}$ & {$[\%]$} & 2.88 & 3.07 & 2.63 & 1.60 & 7.18 & 5.76 & 0.00 & 0.49 & 1.58 \\
$\Delta \eta_{H C}$ & {$[\%]$} & -2.46 & 2.44 & 3.04 & 0.10 & 6.47 & -2.46 & 0.00 & -3.03 & 1.16 \\
$\Delta \eta_{C O}$ & {$[\%]$} & 0.85 & 1.37 & 1.83 & 1.21 & -3.19 & 0.85 & 0.00 & 3.77 & -1.04 \\
& {$[\%]$} & -0.09 & 0.59 & 0.74 & 0.41 & 0.01 & -0.18 & 0.00 & -0.20 & -0.02
\end{tabular}

In general, the temperature penalty term has a negligible effect for the thermal management control of the TWC. In fact, by looking at the Fig. 4a and Fig. 4b which show the maximum temperature measured at the sampling point $\frac{L}{2}$ for the 9 combinations of $T_{T W C}^{u p}$ and slope factor in the first two driving phases of the WLTC, only in the initial urban driving phase, there is a small difference in the average temperature between the calibrations. Furthermore, also the increase of the slope factor of the penalty term does not have any effect in containing the TWC temperature within the desired temperature operational window $\left[T_{T W C}^{L O}, T_{T W C}^{u p}\right]$. 


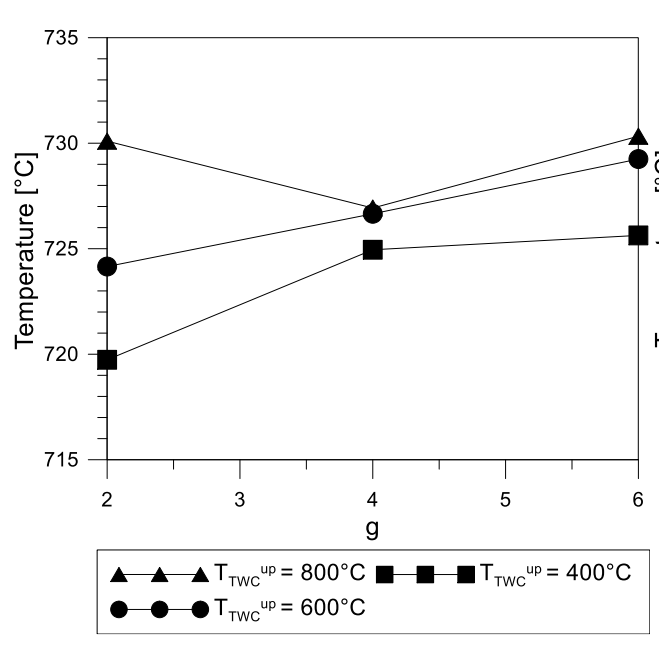

(a)

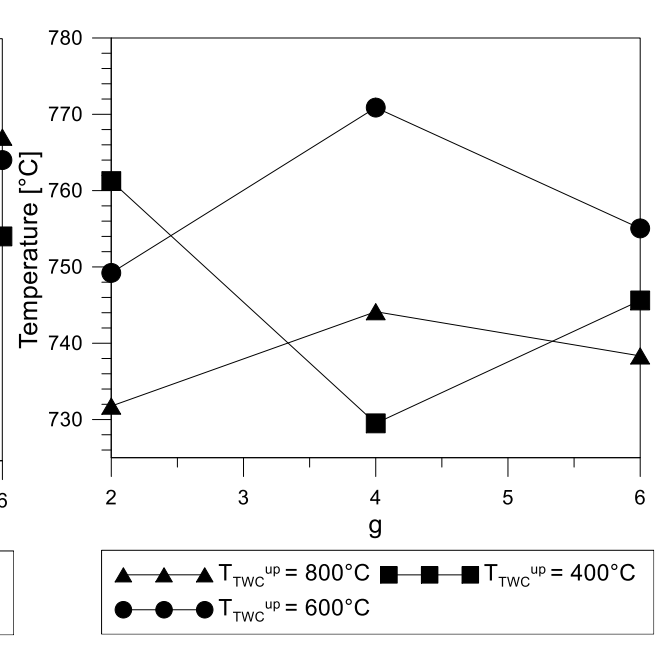

(b)

Fig. 4. Maximum temperature of TWC at the sampling point located at half-length on the axis for different values of $T_{T W C}^{u p}$. Fig. $4 \mathrm{a}$ and Fig. $4 \mathrm{~b}$ respectively report data for the low and medium phases of the WLTC

This behavior can be better explained by looking at the SoC trajectories, plotted in Fig. 5 a only for the cases corresponding to $T_{T W C}^{u p}=600^{\circ} \mathrm{C}$, used in Fig. 4, as an example. The increase in the penalty gradient value $g$ would look like to have a counter effect to the desired one (limiting the TWC temperature within $\left[T_{T W C}^{L O}, T_{T W C}^{u p}\right]$ ), resulting in a greater use of the ICE which recharges the battery in the first half of the driving cycle and simultaneously heats the TWC. However, this behaviour is caused by the charge sustaining condition of the energy management control strategy, that can be maintained only by increasing the constant term of the equivalence factor, $s_{0}$, as it is displayed in Fig. $5 \mathrm{~b}$. The increase of $s_{0}$ value has a positive effect on the ICE usage. In conclusion it can be remarked that the charge sustaining condition and the TWC thermal management control have an opposite effect on the energy management strategy of the hybrid powertrain and this leads to an unsuccessful control of the TWC thermal management by means of calibration of the presented supervisory energy management control strategy based on the a-ECMS algorithm. 


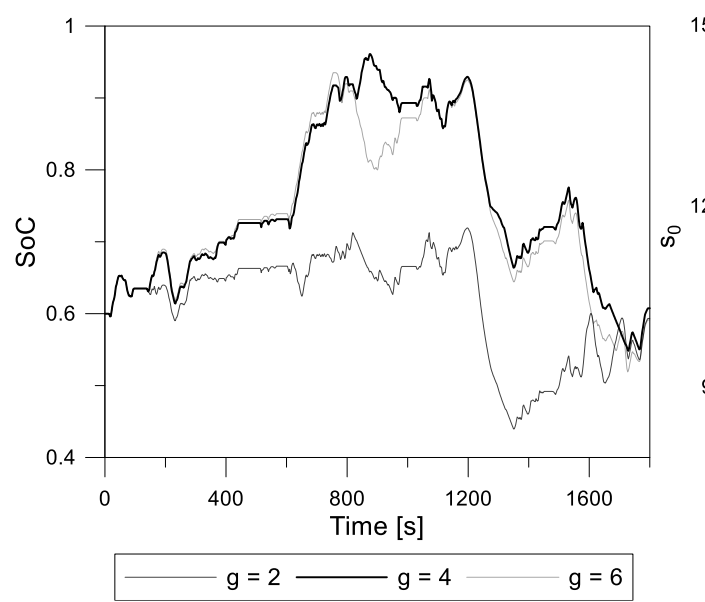

(a)

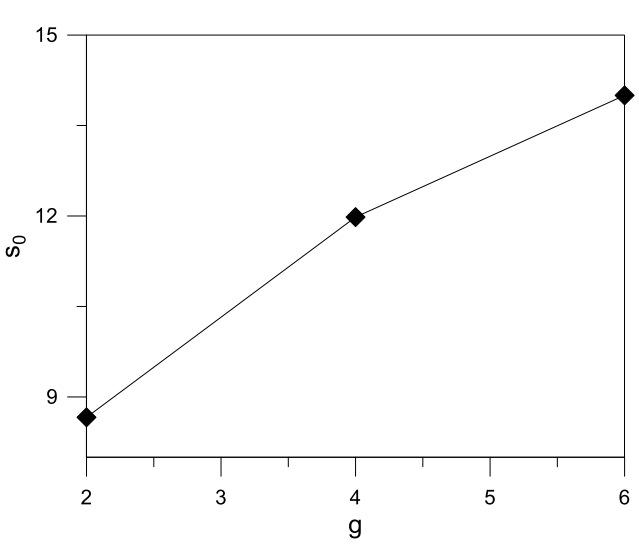

(b)

Fig. 5. (a) Battery state-of-charge trajectories and (b) equivalence factor constant term $s_{0}$ for the cases with $T_{T W C}^{u p}=600^{\circ} \mathrm{C}$

\subsection{Calibration with Additional Temperature Sampling Points}

Two additional temperature sampling points are considered apart from the one used at half of the length of the TWC axis. A preliminary observation must be made to highlight the difference of the three sampling points. The temperature evolution is delayed at an increasing distance from TWC inlet as can be seen from Fig. 6 .

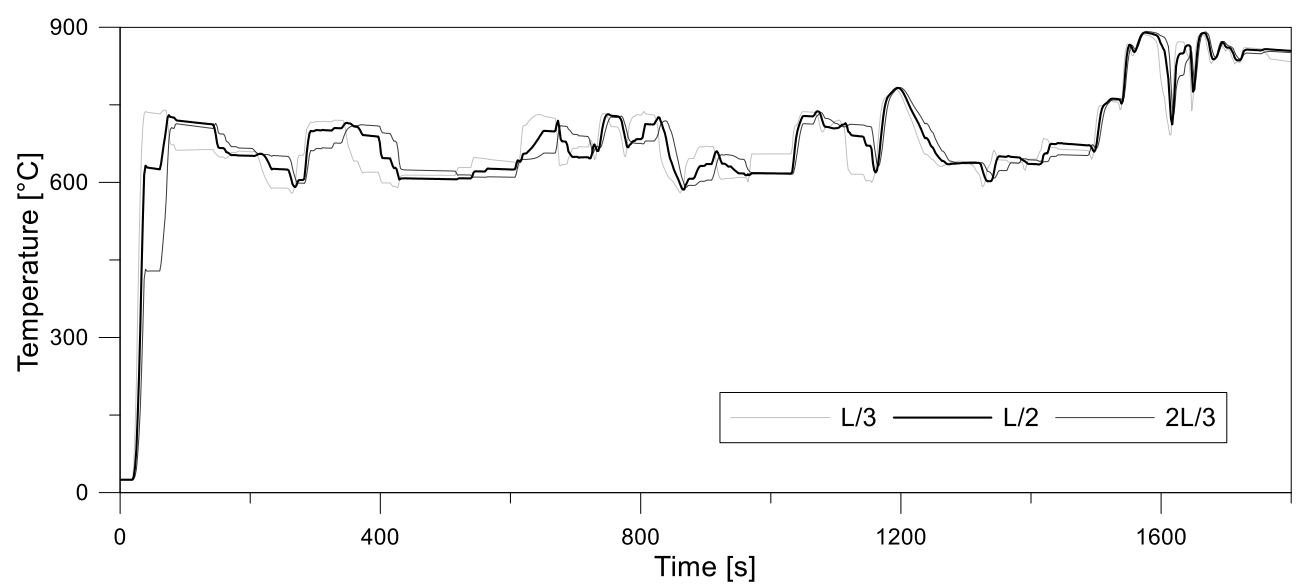

Fig. 6. TWC temperature measured at three sampling points on the axis.

The integration of three temperature sampling points was then also studied for different values of the weight coefficients $w_{1}, w_{2}$ and $w_{3}$. The expected behavior given by a calibration of these weights, so that the one corresponding to the furthest point from the TWC inlet $\left(w_{3}\right)$ is set to a higher value than the one for the closest point to the TWC inlet $\left(w_{1}\right)$, should be to favor a greater use of the ICE, at least in the initial part of the driving cycle, since the weighted-sum temperature is more influenced by the lower one at the extremal point (at $\left.\frac{2 L}{3}\right)$. On the contrary, when $w_{1}>w_{3}$ the effect should be opposite. The results obtained prove this 
concept as showed in Fig. 7, where the total energy output of the ICE is examined for the first phase of the WLTC.

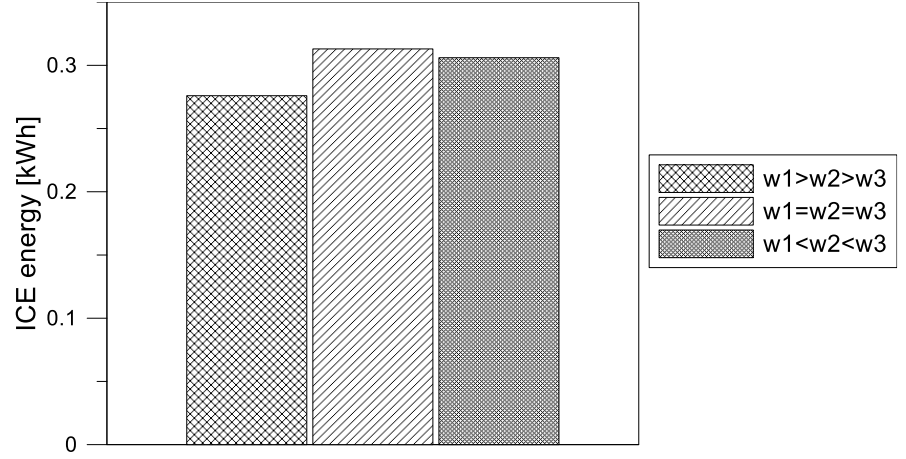

Fig. 7. ICE energy output during the low phase of the WLTC driving cycle for three calibration setups of the weights

Finally, similarly to the previous subsection, results are presented in Table 2. Data is presented as percentage deviation with respect to the baseline case with one TWC temperature sampling point for fuel consumption and TWC conversion efficiencies. As can be inferred from Table 2 , when $w_{1}$ value is higher than $w_{3}$, the maximum temperature value achieved during the low driving phase of the WLTC is lower and this can be justified by the previous explanation about the expected effect of the weights tuning. However, once again the effect is negligible and it is reflected by the fact that fuel consumption increase is in the end very low when using a calibration which should favor a greater use of the ICE $\left(w_{3}>\right.$ $w_{1}$ ). Also, the variation in conversion efficiency for the most relevant polluting species is very small, but this effect is a direct consequence of how the TWC temperature varies over time.

Table 2. Characteristic results of the TWC performance for different calibrations of the weights. Fuel consumption and conversion efficiencies are referred to the case highlighted in bold

\begin{tabular}{c|c|c|c|c|c}
\multicolumn{1}{c|}{$T_{T W C}^{u p}$} & {$\left[{ }^{\circ} \mathrm{C}\right]$} & $\begin{array}{c}1 \text { sampling } \\
\text { point }\end{array}$ & $w_{1}>w_{2}>w_{3}$ & $w_{1}=w_{2}=w_{3}$ & $w_{1}<w_{2}<w_{3}$ \\
\hline$\left(T_{T W C}^{\max }\right)_{W L T C-l o w}$ & {$\left[{ }^{\circ} \mathrm{C}\right]$} & 730 & 723 & 726 & 727 \\
$\left(T_{T W C}^{\text {max }}\right)_{W L T C-\text { medium }}$ & {$\left[{ }^{\circ} \mathrm{C}\right]$} & 732 & 734 & 750 & 730 \\
Time to $T_{T W C}^{L O}$ & {$[\mathrm{~s}]$} & 31 & 31 & 31 & 31 \\
$\Delta F C$ & {$[\%]$} & 0.00 & 0.90 & 1.61 & 1.70 \\
$\Delta \eta_{N O}$ & {$[\%]$} & 0.00 & 2.80 & 2.44 & 3.16 \\
$\Delta \eta_{H C}$ & {$[\%]$} & 0.00 & 2.25 & 3.05 & 1.35 \\
$\Delta \eta_{C O}$ & {$[\%]$} & 0.00 & 0.70 & 1.14 & 1.14
\end{tabular}

To better clarify this point, the temperature evolution at the three sampling points is reported in Fig. 8. It can be seen how the impact of the different tunings on the TWC thermal management is negligible. The TWC behavior is strictly dependent on how the energy management strategy operates the power-split control between the ICE and EM, which must satisfy the charge sustaining condition. 


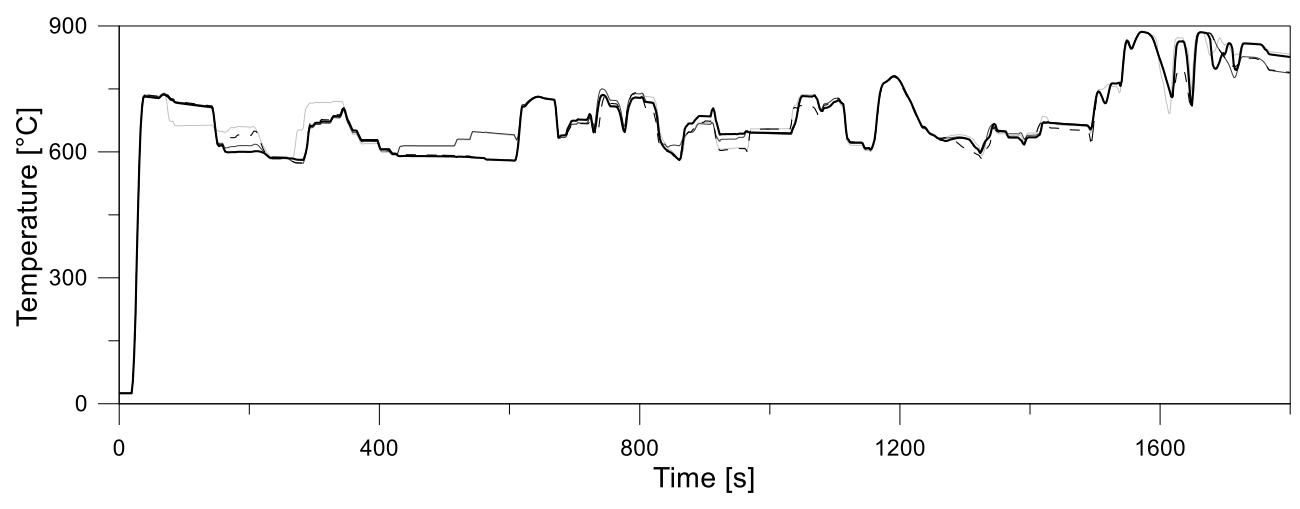

(a)

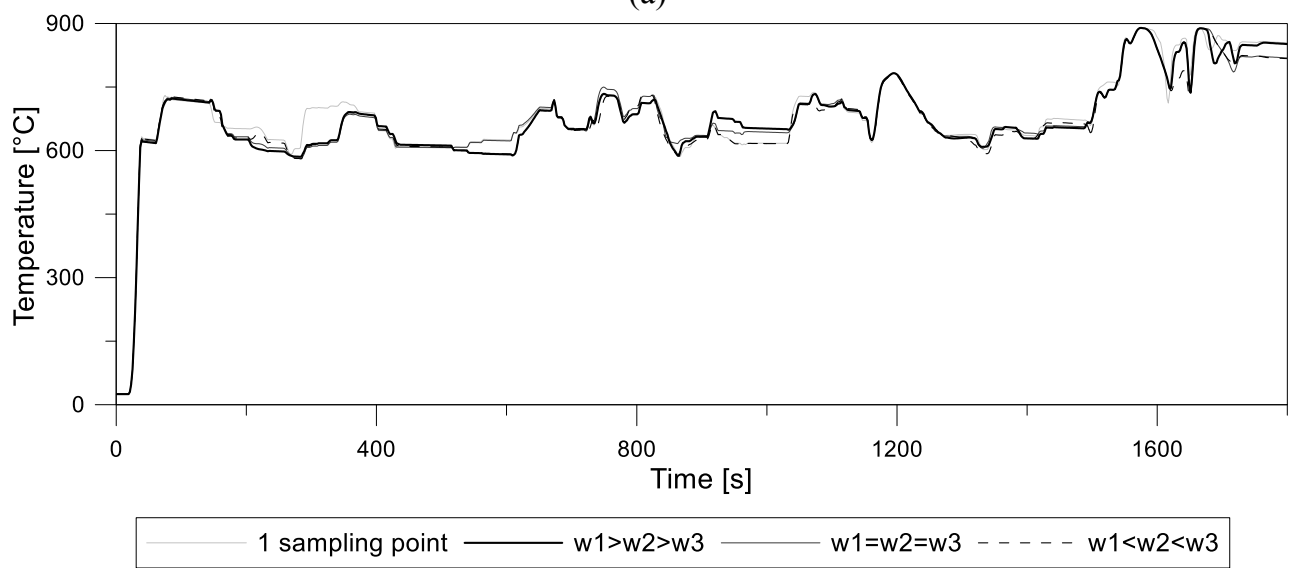

(b)

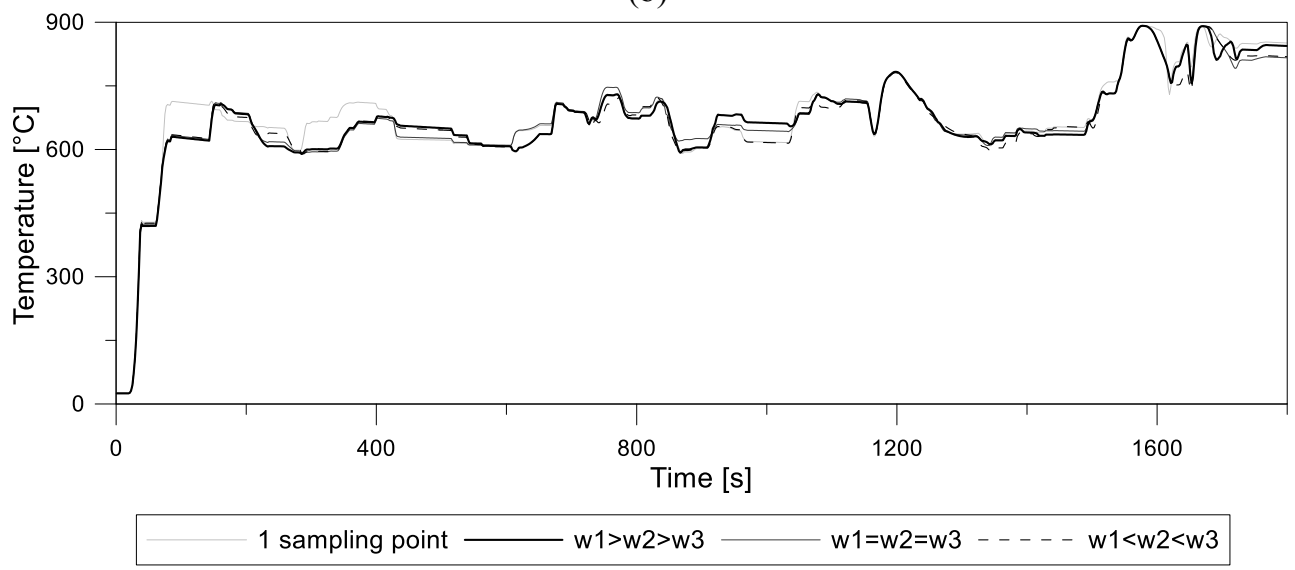

(c)

Fig. 8. TWC temperature at the three sampling points (a) $\mathrm{L} / 3$, (b) $\mathrm{L} / 2$, (c) $2 \mathrm{~L} / 3$ for different calibrations of the weights 


\section{Conclusions}

The three-way catalyst thermal management is an important aspect to take into account in the design of the supervisory energy management strategy for a gasoline hybrid electric vehicle, especially to mitigate the inefficiency of the catalyst during the cold start. This study analyzes different calibration setups of a supervisory energy management strategy, based on an Adaptive Equivalent Consumption Minimization Strategy algorithm, which integrates the three-way catalyst thermal management. Under the assumption that the engine is operated with a lambda close to 1 , the fundamental parameter to determine the catalyst conversion efficiency is its temperature. Therefore, different configurations of the cost function were tested to analyze the impact of the developed supervisory energy management strategy on the three-way catalyst thermal management. In particular, two aspects were evaluated: different tunings of the penalty term used to set the catalyst operating temperature window; the influence of additional temperature sampling points with respect to only one on the catalyst axis midspan.

The investigation, which was carried out through numerical simulations, shows that the thermal management of the three way catalyst requires the use of the internal combustion engine to warm up the catalyst and to reduce the time to the light-off temperature in the early phase of the driving cycle. Moreover, the three-way catalyst operational temperature could be maintained below the limit temperature (i.e. $800^{\circ} \mathrm{C}$ ), which is beyond the catalyst optimal operating temperature, so to prevent a fast aging. The results obtained through numerical simulation show that this aspect was not adequately fulfilled by the presented control strategy, even though different setups of the three-way catalyst temperature penalty term were tested. In fact, when looking at the first phase of the WLTC driving cycle, as the penalty factor for the catalyst temperature was increased, there was only a decrease of almost 10 degrees in the maximum temperature of the catalyst. This outcome may be addressed to the level of electrification of the powertrain since it cannot run under pure electric mode for prolonged time, consequently leading to a continuous use of the engine and also to the constraining condition to maintain the battery state of charge within the range (0.4-0.8), which must however be satisfied to preserve battery life. Furthermore, exploiting the onedimensional model of the three-way catalyst by adding two temperature sampling points along the catalyst axis, at $1 / 3$ and $2 / 3$ of its length, had a negligible impact on improving the thermal management of the catalytic converter.

It can be concluded that as the charge sustaining condition must be satisfied, a proper calibration of the supervisory energy management control strategy fails to simultaneously satisfy the imposed condition for the three-way catalyst as it is expressed as a soft penalty.

\section{Definitions/Abbreviations}

BMS - Battery Management System

DP - Dynamic Programming

ECU - Engine Control Unit

ECMS - Equivalent Consumption Minimization Strategy

EM - Electric Machine

FHEV - Full Hybrid Electric Vehicle

ICE - Internal Combustion Engine

SoC - State of Charge

TWC - Three-way catalyst

WLTC - World-wide harmonized Light-duty Test Cycle 


\section{References}

[1] Hofmann J, Guan D, Chalvatzis K, Huo H. Assessment of electrical vehicles as a successful driver for reducing CO2 emissions in China. Appl Energy 2016;184:9951003. https://doi.org/10.1016/j.apenergy.2016.06.042.

[2] Palmer K, Tate JE, Wadud Z, Nellthorp J. Total cost of ownership and market share for hybrid and electric vehicles in the UK, US and Japan. Appl Energy 2018;209:108-19. https://doi.org/10.1016/j.apenergy.2017.10.089.

[3] Senecal PK, Leach F. Diversity in transportation: Why a mix of propulsion technologies is the way forward for the future fleet. Results Eng 2019;4:100060. https://doi.org/10.1016/j.rineng.2019.100060.

[4] Wu G, Zhang X, Dong Z. Powertrain architectures of electrified vehicles: Review, classification and comparison. J Franklin Inst 2015;352:425-48. https://doi.org/10.1016/j.jfranklin.2014.04.018.

[5] Zhuang W, Eben SL. A survey of powertrain con fi guration studies on hybrid electric vehicles 2020;262.

[6] Onori S, Serrao L. On Adaptive-ECMS strategies for hybrid electric vehicles 2011:1-7.

[7] Michel P, Charlet A, Colin G, Chamaillard Y, Bloch G, Nouillant C. Catalytic converter modeling for optimal gasoline-HEV energy management. IFAC Proc Vol 2014;19:6636-41. https://doi.org/10.3182/20140824-6-za-1003.01419.

[8] Guille A, Jeanneret B, Kéromnès A, Le L, Pélissier S. Energy management strategy to reduce pollutant emissions during the catalyst light-off of parallel hybrid vehicles 2020;266.

[9] Benegiamo M, Valletta A, Carlucci P. A, Mulone V. Impact of Thermal Management of the Three-Way Catalyst on the Energy Efficiency of a P2 Gasoline FHEV, SAE Technical Paper 2020-37-0019, 2020.

[10] Ramanathan K, Sharma CS. Kinetic parameters estimation for three way catalyst modeling. Ind Eng Chem Res 2011;50:9960-79. https://doi.org/10.1021/ie200726j.

[11] Paganelli G. General supervisory control policy for the energy optimization of charge-sustaining hybrid electric vehicles. JSAE Rev 2001;22:511-8. https://doi.org/10.1016/s0389-4304(01)00138-2.

[12] Sundstrom O, Guzzella L. A generic dynamic programming Matlab function. 2009 IEEE Int. Conf. Control Appl., IEEE; 2009, p. 1625-30. https://doi.org/10.1109/CCA.2009.5281131.

[13] Ambuhl D. ENERGY MANAGEMENT STRATEGIES FOR HYBRID ELECTRIC VEHICLES presented by. ETH ZURICH, 2009.

[14] Lee S, Bae C, Lee Y, Han T. Effects of engine operating conditions on catalytic converter temperature in an SI engine. SAE Tech Pap 2002. https://doi.org/10.4271/2002-01-1677. 